\title{
Transport Properties of Solitons
}

\author{
A. H. Castro Neto \\ Department of Physics, University of Illinois at Urbana-Champaign \\ 1100 W.Green St., Urbana, IL, 61801-3080, U.S.A. \\ A. O. Caldeira \\ Instituto de Física "Gleb Wataghin" \\ Departamento de Física do Estado Sólido e Ciência dos Materiais \\ Universidade Estadual de Campinas \\ 13081, Campinas, SP, Brazil
}

\begin{abstract}
We calculate in this article the transport coefficients which characterize the dynamics of solitons in quantum field theory using the methods of dissipative quantum systems. We show how the damping and diffusion coefficients of soliton-like excitations can be calculated using the integral functional formalism. The model obtained in this article has new features which cannot be obtained in the standard models of dissipation in quantum mechanics.
\end{abstract}

PACS numbers: 11.10.-z, 11.10.Lm, 03.70.+k, 05.40.+j, 05.60.+w, 03.65.Db 


\section{INTRODUCTION}

There are still controversies about the calculation of transport properties involving soliton-like particles (see [1] and references therein). It is not completely clear how the thermalization of collective excitations, such as solitons, can be carried out. In general, the pathway is based on heuristic approaches which take into account averages over configurations without going deeply inside the dynamical characteristics of the formation of a collective excitation.

The main goal of this article is to link two fields of study which seem to be far apart up to now but, as we shall show, are really closely related.

One of these areas is the quantum theory of solitons developed in the seventies [2] which teaches us how to quantize classical theories which have solitons as solutions of their equations of motion. The other area is the field of dissipative quantum systems which had a great development during the eighties [3].

When we study the dynamics of a classical particle moving through a viscous environment we learn that one can describe its motion using two parameters, the damping and the diffusion coefficient. The former is related to the systematic force applied on the particle by the environment and the latter is related with the fluctuations due to the interaction (for a clear discussion about this issue see [4]).

It was shown some years ago that the same physical situation occurs in quantum systems [3], therefore if a particle interacts with an environment such as a set of decoupled harmonic oscillators (the oscillator model) its motion is damped, which means in quantum mechanical terms that the center of motion of its wavepacket undergoes a damped motion while its width increases with time. Exactly as in the classical problem all the transport properties of the quantum system can be described by a damping and a diffusion coefficient.

One of the main results of the standard approach is that the damping time is independent of temperature and even at zero temperature the motion of the particle is damped. As will show in this article this behaviour is not expect for solitons since the soliton and the 
environment have the same microscopic origin.

From the point of view of quantum field theory, classical and translational invariant theories with solitons can be quantized by the collective coordinate method [2]. In the classical theory, solitons are field configurations which move in space without changing its shape. Therefore, the knowledge of the position of the center of soliton as a function of time is enough to describe its dynamics. In quantum field theory the center of the soliton plays a special role. The center of the soliton can be viewed as a true quantum dynamical variable and we describe the soliton as a particle. The problem here is that at finite temperatures not all the degrees of freedom (infinite, indeed) collaborated in the formation of the soliton and therefore this soliton is never free to move as in the classical theory, that is, there is always a residual interaction due to quantum degrees of freedom which changes drastically the dynamical properties of the system. Our task is to show that, under some approximations, this residual interaction gives rise to a damped motion of the soliton, in other words, the soliton undergoes a brownian motion.

Form this point of view we are presenting here a new model for dissipation in quantum mechanics and, as we would expect, very different from the oscillator model described before.

We could have started from the definition of the model [5], which by itself is completely new in the field of quantum dissipation, without mentioning its origin in quantum field theory, but we think it is of interest to show the generality of the model in this context.

We will show here how we can calculate the transport properties in this framework, namely, the damping and the diffusion coefficient. We will show that the damping time depends on the temperature, a result which can not be obtained in the oscillator model.

In section II we present the results of the quantization of the solitons in field theory and the approximations involved in our model. Although the same approach can be found in great detail in Ref. [2] we include this topic here for the sake of completeness. In Section III we develop the the integral functional formalism which allows us to calculate the transport properties. Section IV contains our conclusions. 


\section{QUANTIZATION OF SOLITONS}

Let us consider a classical action in $1+1$ dimensions for a scalar field,

$$
S=\int d^{2} x\left(\frac{1}{2}\left(\partial^{\mu} \phi\right)\left(\partial_{\mu} \phi\right)-U(g, \phi)\right)
$$

where $U$ is a potential function of the field $\phi$ and the coupling constant $g$. Here we will assume that $U$ has a degenerate absolute minimum. We can always rescale the field such as $\phi \rightarrow \phi / g$ and the potential as $U \rightarrow U / g^{2}$ (by naive dimensional analysis) and rewrite (2.1) as

$$
S=\frac{1}{g^{2}} \int d^{2} x\left(\frac{1}{2}\left(\partial^{\mu} \phi\right)\left(\partial_{\mu} \phi\right)-U(\phi)\right)
$$

Consider the static part of (2.2),

$$
V=\frac{1}{g^{2}} \int d^{2} x\left(\frac{1}{2}\left(\frac{\partial \phi}{\partial x}\right)^{2}+U(\phi)\right)
$$

and its extremum value given by $\delta V / \delta \phi_{s}=0$, namely,

$$
\frac{d^{2} \phi_{s}}{d x^{2}}=\frac{d U\left(\phi_{s}\right)}{d \phi_{s}}
$$

which, due to the translational invariance of (2.1), must have the form

$$
\phi_{s}=\phi_{s}\left(x-x_{0}\right)
$$

where $x_{0}$ is a constant. Eq.(2.5) is a static localized solution of the classical equations of motion.

We expand (2.3) around the extremum, (2.4), in terms of the coupling constant $g$ as,

$$
\phi(x, t)=\phi_{s}\left(x-x_{0}\right)+g \delta \phi(x, t)
$$

and one finds,

$$
V[\delta \phi]=V\left[\phi_{s}\right]+\int d^{2} x \delta \phi(x, t)\left(-\frac{d^{2}}{d x^{2}}+\left(\frac{d^{2} U}{d \phi^{2}}\right)_{\phi_{s}}\right) \delta \phi(x, t)
$$

plus higher order terms in $g$. 
Using (2.4) we get,

$$
M=V\left[\phi_{s}\right]=\frac{1}{g^{2}} \int d^{2} x\left(\frac{d \phi_{s}}{d x}\right)^{2}
$$

is the classical soliton mass.

Therefore, up to leading order in $g$, the eigenmodes of the system are given by

$$
\left(-\frac{d^{2}}{d x^{2}}+\left(\frac{d^{2} U}{d \phi^{2}}\right)_{\phi_{s}}\right) \Psi_{n}\left(x-x_{0}\right)=\Omega_{n}^{2} \Psi_{n}\left(x-x_{0}\right) .
$$

Observe that the function $d \phi_{s} / d x$ is an eigenfunction with eigenvalue zero (use (2.4) in (2.9)) indicating that we should expect divergencies in high order terms in the perturbative expansion. The way to deal with this "zero" mode is the following: suppose we displace the center of the soliton, $x_{0}$, by an infinitesimal amount $\delta x_{0}$. Thus, up to first order in $\delta x_{0}$,

$$
\delta_{0} \phi_{s}=\phi_{s}\left(x_{0}+\delta x_{0}\right)-\phi_{s}\left(x_{0}\right)=\frac{\partial \phi_{s}}{\partial x_{0}} \delta x_{0}
$$

but since $\phi_{s}$ is a function of the difference $x-x_{0}$ we can rewrite (2.10) as

$$
\delta_{0} \phi_{s}=-\frac{d \phi_{s}}{d x} \delta x_{0}
$$

therefore the eigenmode $d \phi_{s} / d x$ is related with the movement of the localized solution, that is, the motion of the soliton. Call,

$$
\Psi_{0}\left(x-x_{0}\right)=\frac{1}{N} \frac{d \phi_{s}}{d x}
$$

where $N$ is a normalization constant.

We rewrite $\delta \phi$ in $(2.6)$ as,

$$
\delta \phi(x, t)=\sum_{n=0}^{\infty} a_{n}(t) \Psi_{n}\left(x-x_{0}\right) .
$$

Therefore, from (2.6),

$$
\phi(x, t)=\phi_{s}\left(x-x_{0}\right)+\frac{g}{N} a_{0}(t) \frac{d \phi_{s}}{d x}+g \sum_{n=1}^{\infty} a_{n}(t) \Psi_{n}\left(x-x_{0}\right) .
$$

Notice that the first two terms in r.h.s. of (2.14) appear in the expansion obtained in (2.10). In this way we see that the center of the soliton is a true dynamical variable and we rewrite the expansion (2.14) as, 


$$
\phi(x, t)=\phi_{s}\left(x-x_{0}(t)\right)+g \sum_{n=1}^{\infty} a_{n}(t) \Psi\left(x-x_{0}(t)\right)
$$

which is known as the collective coordinate method. All the physics of this system is present in (2.15). The soliton, described by the motion of its center, is a collective excitation (since is a solution of the field equation) but it is coupled to all other modes by the relative coordinate $x-x_{0}$.

We can now rewrite the action (2.2) in the presence of the soliton using (2.14) (see [2] for details) and obtain the classical Hamiltonian for his problem,

$$
H=-M+\frac{1}{2 M}\left(P-\sum_{n, m=1}^{\infty} G_{m n} p_{n} q_{m}\right)^{2}+\sum_{n=1}^{\infty}\left(\frac{p_{n}^{2}}{2}+\frac{\Omega_{n}^{2} q_{n}^{2}}{2}\right)
$$

plus higher order terms in $g . P$ is the momentum canonically conjugated to $x_{0}, q_{n}$ and $p_{n}$ are the conjugated pairs for the modes and

$$
G_{m n}=-\int d x \frac{d \Psi_{m}}{d x}(x) \Psi_{n}(x)
$$

couples the soliton to the other modes (mesons) in the system.

It is clear now that the soliton can not move freely, the mesons are scattered by the soliton which will move as a brownian particle. Although (2.16) is perturbative in the coupling constant, we will assume that the main physics of the problem is present in that expression.

The quantization of (2.16) is straightforward. We impose the commutation relations

$$
\begin{gathered}
{\left[\hat{x}_{0}, \hat{P}\right]=i \hbar} \\
{\left[\hat{q}_{n}, \hat{p}_{m}\right]=i \hbar \delta_{n m}}
\end{gathered}
$$

and write (2.16) in the symmetrized form (apart from the constant mass term)

$$
H=\frac{1}{2 M}\left(\hat{P}-\hat{P}_{m e}\right)^{2}+\sum_{n=1}^{\infty} \hbar \Omega_{n} \hat{b}_{n}^{\dagger} \hat{b}_{n}
$$

where 


$$
\hat{b}_{n}=\left(\frac{\Omega_{n}}{2 \hbar}\right)\left(\hat{q}_{n}+i \frac{\hat{p}_{n}}{\Omega_{n}}\right)
$$

which obeys the usual commutation relations for bosons,

$$
\left[\hat{b}_{n}, \hat{b}_{m}^{\dagger}\right]=\delta_{n m} ;\left[\hat{b}_{n}, \hat{b}_{m}\right]=0
$$

In $(2.20), \hat{P}_{m e}$ is the momentum of the mesonic field which is given by

$$
\hat{P}_{m e}=\hat{P}_{d}+\hat{P}_{n d}
$$

where

$$
\hat{P}_{d}=\sum_{n, m=1}^{\infty} \frac{\hbar}{2 i}\left(\left(\frac{\Omega_{n}}{\Omega_{m}}\right)^{1 / 2}+\left(\frac{\Omega_{m}}{\Omega_{n}}\right)^{1 / 2}\right) G_{m n} \hat{b}_{m}^{\dagger} \hat{b}_{n}
$$

and

$$
\hat{P}_{n d}=\sum_{n, m=1}^{\infty} \frac{\hbar}{4 i}\left(\left(\frac{\Omega_{n}}{\Omega_{m}}\right)^{1 / 2}-\left(\frac{\Omega_{m}}{\Omega_{n}}\right)^{1 / 2}\right) G_{m n}\left(\hat{b}_{m} \hat{b}_{n}-\hat{b}_{m}^{\dagger} \hat{b}_{n}^{\dagger}\right) .
$$

The Hamiltonian (2.20) is well known in works on polaron dynamics [6,7] and many perturbative approaches has been used in order to understand its physical contents.

Observe that the $P_{n d}$ does not commute with the total number of mesons

$$
\hat{N}=\sum_{n=1}^{\infty} \hat{b}_{n}^{\dagger} \hat{b}_{n}
$$

and therefore it breaks the number conservation. Actually this term is related with high frequency oscillations in which we are not interested. Our task is to show that in the sector of the Hilbert space where the number $\hat{N}$ is conserved the physics of $(2.20)$ is that of a brownian particle. Therefore our new model in the context of quantum dissipation is based on the following Hamiltonian [5]

$$
H=\frac{1}{2 M}:\left(\hat{P}-\sum_{n, m=1}^{\infty} \hbar g_{m n} \hat{b}_{n}^{\dagger} \hat{b}_{n}\right)^{2}:+\sum_{n=1}^{\infty} \hbar \Omega_{n} \hat{b}_{n}^{\dagger} \hat{b}_{n}
$$

where : ... : means normal order and

$$
g_{m n}=\frac{1}{2 i}\left(\left(\frac{\Omega_{n}}{\Omega_{m}}\right)^{1 / 2}+\left(\frac{\Omega_{m}}{\Omega_{n}}\right)^{1 / 2}\right) G_{m n}
$$


is the coupling constant of the theory.

We believe that Hamiltonian (2.27) describes the main physics of the dynamics of solitons at low energies and, by itself, can be used to model other physical systems where the coupling of the particle and the environment can not be described by the standard model.

\section{FUNCTIONAL INTEGRAL METHOD}

The starting point for the calculations of the transport properties of the soliton is the well-known Feynman-Vernon formalism [8] that the authors have recently applied [5] to the Hamiltonian (2.27).

We are interested only in the quantum statistical properties of the soliton and the mesons act only as a source of relaxation and diffusion processes. Consider the density operator for the system soliton plus mesons, $\hat{\rho}(t)$. This operator evolves in time according to

$$
\hat{\rho}(t)=e^{-i \hat{H} t / \hbar} \hat{\rho}(0) e^{i \hat{H} t / \hbar}
$$

where $\hat{H}$ is given by $(2.27)$ and $\hat{\rho}(0)$ is the density operator at $t=0$ which we will assume to be decoupled as a product of the soliton density operator, $\hat{\rho}_{S}(0)$, and the meson density operator, $\hat{\rho}_{R}(0)$,

$$
\hat{\rho}(0)=\hat{\rho}_{S}(0) \hat{\rho}_{R}(0)
$$

where the symbol $\underline{S}$ refers to the soliton (system of interest) and $\underline{R}$ to the mesons (the reservoir of excitations).

We will consider that the mesons are in thermal equilibrium at $t=0$, that is,

$$
\hat{\rho}_{R}(0)=\frac{e^{-\beta \hat{H}_{R}}}{Z}
$$

where

$$
Z=\operatorname{tr}_{R}\left(e^{-\beta \hat{H}_{R}}\right)
$$

with 


$$
\beta=\frac{1}{K_{B} T}
$$

Here $\operatorname{tr}_{R}$ denotes the trace over the mesons variables and $K_{B}$ is the Boltzmann constant. $\hat{H}_{B}$ is the free meson Hamiltonian which is given by the last term on the right hand side of (2.27).

As we said, we are interested only in the quantum dynamics of the system S, so, we define a reduced density operator

$$
\hat{\rho}_{s}(t)=\operatorname{tr}_{R}(\hat{\rho}(t))
$$

which contains all the information about $\underline{S}$ when it is in contact with $\underline{R}$.

Projecting now (3.1) in the coordinate representation of the soliton system 9]

$$
\hat{x}_{o}|q>=q| q>
$$

and in the coherent state representation for bosons (the mesons),

$$
\hat{b}_{n}\left|\alpha_{n}>=\alpha_{n}\right| \alpha_{n}>
$$

we have [11] (see ref. [0] for details)

$$
\rho_{s}(x, y, t)=\int d x^{\prime} \int d y^{\prime} J\left(x, y, t ; x^{\prime}, y^{\prime}, 0\right) \rho_{s}\left(x^{\prime}, y^{\prime}, 0\right)
$$

Here we have used (3.2), (3.3), (3.6) and the completeness relation for the representations above, namely,

$$
\begin{gathered}
\int d q|q><q|=1 \\
\int \frac{d^{2} \alpha}{\pi}|\alpha><\alpha|=1
\end{gathered}
$$

where $d^{2} \alpha=d(\operatorname{Re} \alpha) d(\operatorname{Im} \alpha)$ as usual.

In (3.9), $J$ is the superpropagator of the soliton, which can be written as

$$
J=\int_{x^{\prime}}^{x} D x \int_{y^{\prime}}^{y} D y e^{\frac{i}{\hbar}\left(S_{o}[x]-S_{o}[y]\right)} F[x, y]
$$


where

$$
S_{o}[x]=\int_{0}^{t} d t^{\prime}\left\{\frac{M_{o} \dot{x}^{2}\left(t^{\prime}\right)}{2}\right\}
$$

is the classical action for the free particle. F is the so-called influence functional,

$$
F[x, y]=\int \frac{d^{2} \vec{\alpha}}{\pi^{N}} \int \frac{d^{2} \vec{\beta}}{\pi^{N}} \int \frac{d^{2} \vec{\beta}^{\prime}}{\pi^{N}} \rho_{R}\left(\vec{\beta}^{*}, \vec{\beta}^{\prime}\right) e^{-|\vec{\alpha}|^{2}-\frac{|\vec{\beta}|^{2}}{2}-\frac{\left|\vec{\beta}^{\prime}\right|^{2}}{2}} \int_{\vec{\beta}}^{\vec{\alpha}^{*}} D^{2} \vec{\alpha} \int_{\vec{\beta}^{\prime *}}^{\vec{\alpha}} D^{2} \vec{\gamma} e^{S_{I}[x, \vec{\alpha}]+S_{I}^{*}[y, \vec{\gamma}]}
$$

where $\vec{\beta}$ denotes the vector $\left(\beta_{1}, \beta_{2}, \beta_{3}, \ldots, \beta_{N}\right)$ and $S_{I}$ is a complex action related to the reservoir plus interaction,

$$
S_{I}[x, \vec{\alpha}]=\int_{0}^{t} d t^{\prime}\left\{\frac{1}{2}\left(\vec{\alpha} \cdot \frac{d \vec{\alpha}^{*}}{d t^{\prime}}-\vec{\alpha}^{*} \cdot \frac{d \vec{\alpha}}{d t^{\prime}}\right)-\frac{i}{\hbar}\left(H_{R}-\dot{x} h_{I}\right)\right\}
$$

with

$$
\begin{gathered}
H_{R}=\sum_{n=1}^{\infty} \hbar \Omega_{n} \alpha_{n}^{*} \alpha_{n} \\
h_{I}=\sum_{n, m=1}^{\infty} \hbar g_{n m} \alpha_{m}^{*} \alpha_{n} .
\end{gathered}
$$

In our case the lagrangean formalism simplifies the problem transforming a nonlinear problem into a linear one. The action (3.15) is quadratic in $\vec{\alpha}$, so it can be solved exactly. Observe that the Euler-Lagrange equations for (3.15) are

$$
\begin{aligned}
& \dot{\alpha}_{n}+i \Omega_{n} \alpha_{n}-i \dot{x} \sum_{m=1}^{\infty} g_{m n} \alpha_{m}=0 \\
& \dot{\alpha}_{n}^{*}-i \Omega_{n} \alpha_{n}^{*}+i \dot{x} \sum_{m=1}^{\infty} g_{n m} \alpha_{m}^{*}=0
\end{aligned}
$$

which must be solved subject to the boundary conditions

$$
\begin{gathered}
\alpha_{n}(0)=\beta_{n} \\
\alpha_{n}^{*}(t)=\alpha_{n}^{*} .
\end{gathered}
$$


Due to (2.17) we have $g_{n n}=0$ and the modes are not coupled among themselves. This makes (3.18) and (3.19) easy to solve. That set of equations represents a set of harmonic oscillators forced by the presence of the soliton. The result can be written as,

$$
\begin{gathered}
\alpha_{n}(\tau)=e^{-i \Omega_{n} \tau}\left(\beta_{n}+\sum_{m=1}^{\infty} W_{n m}(\tau) \beta_{m}\right) \\
\alpha_{n}^{*}(\tau)=e^{i \Omega_{n} \tau}\left(\alpha_{n}^{*} e^{-i \Omega_{n} t}+\sum_{m=1}^{\infty} \tilde{W}_{n m}(\tau) e^{-i \Omega_{m} t} \alpha_{m}^{*}\right)
\end{gathered}
$$

where $W_{n m}$ and $\tilde{W}_{n m}$ are functionals of $x(t)$ which obey the following equations

$$
\begin{aligned}
& W_{n m}(\tau)=\delta_{n m}+\sum_{n^{\prime}=1}^{\infty} \int_{0}^{\tau} d t^{\prime} K_{n n^{\prime}}\left(t^{\prime}\right) W_{n^{\prime} m}\left(t^{\prime}\right) \\
& \tilde{W}_{n m}(\tau)=\delta_{n m}+\sum_{n^{\prime}=1}^{\infty} \int_{\tau}^{t} d t^{\prime} K_{n n^{\prime}}\left(t^{\prime}\right) \tilde{W}_{n^{\prime} m}\left(t^{\prime}\right)
\end{aligned}
$$

where

$$
K_{n m}([x], \tau)=i g_{n m} \dot{x}\left(t^{\prime}\right) e^{i\left(\Omega_{n}-\Omega_{m}\right) t^{\prime}}
$$

is the kernel of the integral equation (observe that: $W_{n m}(t)=\tilde{W}_{m n}(0)$ ).

Now we expand the action (3.15) around the classical solution (3.22) and (3.23) and obtain, after some integrations in (3.14)

$$
F[x, y]=\prod_{n=1}^{\infty}\left(1-\Gamma_{n n}[x, y] \bar{n}_{n}\right)^{-1}
$$

where

$$
\Gamma_{n m}=W_{n m}^{*}[y]+W_{m n}[x]+\sum_{\ell=1}^{\infty} W_{\ell m}^{*}[y] W_{\ell n}[x]
$$

with

$$
\bar{n}_{n}=\left(e^{\beta \hbar \Omega_{n}}-1\right)^{-1}
$$

Notice that (3.27) and (3.28) are exact, no approximations have been made so far. 
We see from (3.24) and (3.26) that $W_{n m}$ can be expressed as a power series expansion of the Fourier transform of the soliton velocity, $\dot{x}$, so for small soliton velocities we expect that only few terms in (3.24) will be sufficient for a good description of the soliton dynamics.

Another way to see this is to notice that (3.24) and (3.25) are the scattering amplitudes from the mode $k$ to the mode $j$. The terms that appear in the sum represent the virtual transitions between these two modes. With these two arguments in mind we will make use of the Born-approximation. In matrix notation,

$$
W=\left(1-W^{o}\right)^{-1} W^{o} \simeq W^{o}+W^{o} W^{o}
$$

Therefore, in the approximation of small soliton velocity the terms in (3.28) are small and we can rewrite as a good approximation

$$
F[x, y] \simeq \exp \left\{\sum_{n=1}^{\infty} \Gamma_{n n}[x, y] \bar{n}_{n}\right\} .
$$

Observe that if the interaction is turned off $(\Gamma \rightarrow 0)$ or the temperature is zero $(T=0)$ the functional (3.31) is one, and, as we would expect the soliton moves as a free particle.

Substituting the Born approximation (3.30) in (3.31) and the latter in (3.12) we find

$$
J=\int_{x^{\prime}}^{x} D x \int_{y^{\prime}}^{y} D y \exp \left\{\frac{i}{\hbar} \tilde{S}[x, y]+\frac{1}{\hbar} \tilde{\phi}[x, y]\right\}
$$

where

$$
\tilde{S}=\int_{0}^{t} d t^{\prime}\left\{\frac{M_{o}}{2}\left(\dot{x}^{2}\left(t^{\prime}\right)-\dot{y}^{2}\left(t^{\prime}\right)\right)+\left(\dot{x}\left(t^{\prime}\right)-\dot{y}\left(t^{\prime}\right)\right) \int_{0}^{t} d t^{\prime \prime} \Gamma_{I}\left(t^{\prime}-t^{\prime \prime}\right)\left(\dot{x}\left(t^{\prime \prime}\right)+\dot{y}\left(t^{\prime \prime}\right)\right)\right\}
$$

and

$$
\tilde{\phi}=\int_{0}^{t} d t^{\prime} \int_{0}^{t} d t^{\prime \prime}\left\{\Gamma_{R}\left(t^{\prime}-t^{\prime \prime}\right)\left(\dot{x}\left(t^{\prime}\right)-\dot{y}\left(t^{\prime}\right)\right)\left(\dot{x}\left(t^{\prime \prime}\right)-\dot{y}\left(t^{\prime \prime}\right)\right)\right\}
$$

with

$$
\begin{aligned}
& \Gamma_{R}(t)=\hbar \theta(t) \sum_{n, m=1}^{\infty} g_{n m}^{2} \bar{n}_{n} \cos \left(\Omega_{n}-\Omega_{m}\right) t \\
& \Gamma_{I}(t)=\hbar \theta(t) \sum_{n, m=1}^{\infty} g_{n m}^{2} \bar{n}_{n} \sin \left(\Omega_{n}-\Omega_{m}\right) t .
\end{aligned}
$$


Now, if we define the new variables $\mathrm{R}$ and $\mathrm{r}$ as,

$$
\begin{aligned}
& R=\frac{x+y}{2} \\
& r=x-y
\end{aligned}
$$

the equations of motion for the action in (3.33) read,

$$
\begin{gathered}
\ddot{R}(\tau)+2 \int_{0}^{t} d t^{\prime} \gamma\left(\tau-t^{\prime}\right) \dot{R}\left(t^{\prime}\right)=0 \\
\ddot{r}(\tau)-2 \int_{0}^{t} d t^{\prime} \gamma\left(t^{\prime}-\tau\right) \dot{r}\left(t^{\prime}\right)=0
\end{gathered}
$$

where

$$
\gamma(t)=\frac{1}{M_{o}} \frac{d \Gamma_{I}}{d t}
$$

or, using (3.36),

$$
\gamma(t)=\frac{\hbar \theta(t)}{M_{o}} \sum_{n, m=1}^{\infty} g_{n m}^{2} \bar{n}_{n}\left(\Omega_{n}-\Omega_{m}\right) \cos \left(\Omega_{n}-\Omega_{m}\right) t
$$

is the damping function.

In terms of these newly defined variables, we can easily see that (3.39) and (3.40) have the same form of the equations previously obtained in the case of quantum brownian motion [3], except for the fact that they now present memory effects (see ref. [3] for details).

Furthermore, in the limit where the time scale of interest is much greater than the correlation time of the meson variables we can write [12],

$$
\gamma(t)=\bar{\gamma}(T) \delta(t)
$$

where $\bar{\gamma}(T)$ is a damping parameter which is temperature dependent and $\delta(t)$ is the Dirac delta function. The form (3.43) is known as the Markovian approximation, because in this case the memory is purely local and does not depend on the previous motion of the soliton.

If we use (3.39) and (3.40) with (3.43) and expand the phase of (3.32) around this classical solution we get the well-known result for the quantum brownian motion [3] where 
the damping parameter $\gamma$ (temperature independent) is replaced by $\bar{\gamma}(T)$ and the diffusive part is replaced by (3.34). As a consequence, the diffusion parameter in momentum space will be given by,

$$
D(t)=\hbar \frac{d^{2} \Gamma_{R}}{d t^{2}}=-\hbar^{2} \theta(t) \sum_{n, m=1}^{\infty} g_{n m}^{2} \bar{n}_{n}\left(\Omega_{n}-\Omega_{m}\right)^{2} \cos \left(\Omega_{n}-\Omega_{m}\right) t
$$

As discussed before, in the Markovian limit, we can write $D(t)$ in the Markovian form,

$$
D(t)=\bar{D}(T) \delta(t)
$$

where $\bar{D}(T)$ and $\bar{\gamma}(T)$ obey the classical fluctuation-dissipation theorem at low temperatures [10].

In what follows we shall define a function $S\left(\omega, \omega^{\prime}\right)$ which will, in analogy to the spectral function $J(\omega)$ of the standard model [3], allows to replace all the summations over $k$ by integrals over frequencies,

$$
S\left(\omega, \omega^{\prime}\right)=\sum_{n, m=1}^{\infty} g_{n m}^{2} \delta\left(\omega-\Omega_{n}\right) \delta\left(\omega^{\prime}-\Omega_{n}\right)
$$

Notice, however, that unlike $J(\omega)$ in [3], this new function $S\left(\omega, \omega^{\prime}\right)$ is related to the scattering of the environmental excitations between states of frequencies $\omega$ and $\omega^{\prime}$ (as seeing from the laboratory frame). Moreover, due to (3.24) it is easy to see that,

$$
S\left(\omega, \omega^{\prime}\right)=S\left(\omega^{\prime}, \omega\right)
$$

We call $S\left(\omega, \omega^{\prime}\right)$ the "scattering function".

Notice that we can rewrite (3.42) and (3.44) as,

$$
\gamma(t)=\frac{\hbar \theta(t)}{2 M_{o}} \int_{0}^{\infty} d \omega \int_{0}^{\infty} d \omega^{\prime} S\left(\omega, \omega^{\prime}\right)\left(\omega-\omega^{\prime}\right)\left(n(\omega)-n\left(\omega^{\prime}\right)\right) \cos \left(\omega-\omega^{\prime}\right) t
$$

and

$$
D(t)=-\frac{\hbar^{2} \theta(t)}{2} \int_{0}^{\infty} d \omega \int_{0}^{\infty} d \omega^{\prime} S\left(\omega-\omega^{\prime}\right)\left(\omega-\omega^{\prime}\right)^{2}\left(n(\omega)+n\left(\omega^{\prime}\right)\right) \cos \left(\omega-\omega^{\prime}\right) t .
$$

Concluding, we have established that the Hamiltonian (2.27) leads to a brownian dynamics, that is, the soliton moves as a particle in a viscous environment where its relaxation and diffusion are due to the scattering of mesons. 


\section{CONCLUSIONS}

In this article we have showed how a new model for the study of dissipation in quantum mechanics can be obtained from the study the solitons in quantum field theory. We showed how the transport properties of solitons can be calculated in a consistent way from the microscopic point of view.

Our results show that solitons move as a brownian particle at low energies due to the scattering of the mesons which are present in the system. These mesons are the residual excitations created by the presence of the soliton. We showed that the damping and the diffusion coefficients for the soliton motion are dependent of the temperature since the mesons must be thermally activated in order to scatter off the soliton. Therefore, at zero temperature the soliton moves freely, but its mobility decreases as the temperature increases (we have showed this explicitly for the case of the polaron motion, see ref. [7]). All these

results can not be obtained from the standard model since the origin of the particle and the environment is distinct is that case.

\section{ACKNOWLEDGMENTS}

A. H. Castro Neto gratefully acknowledges D. K. Campbell and E. Fradkin for useful comments, R. Rajaraman for a critical reading of the manuscript, Conselho Nacional de Desenvolvimento Científico e Tecnológico, CNPq (Brazil), for a scholarship and the Department of Physics of the University of Illinois for support and hospitality. A.O.Caldeira also wishes to acknowledge the partial support from CNPq. 


\section{REFERENCES}

[1] A.S.Davydov, J.Phys.I 1, 1649, (1991).

[2] R.Rajaraman, Solitons and Instantons, (North Holland, Amsterdam, 1982) and references therein.

[3] A.O.Caldeira and A.J.Leggett, Physica 121 A, 587, (1983).

[4] F.Reif, Fundamentals of Statistical and Thermal Physics, (McGraw-Hill, New York, 1965).

[5] A.H.Castro Neto and A.O.Caldeira, Phys.Rev.Lett., 1960, (1991).

[6] T.D.Holstein, Mol.Cryst.Liq.Cryst. 77, 235, (1981); L.A.Turkevich and T.D.Holstein, Phys.Rev.B35, 7474, (1987).

[7] A.H.Castro Neto and A.O.Caldeira, Phys.Rev.B 46, 8858, (1992).

[8] R.P.Feynman and F.L.Vernon, Annals of Physics 24, 118, (1963).

[9] J.Goldstone and R.Jackiw, Phys.Rev. D11, 1486, (1975).

[10] R.Kubo, Rep.Prog.Phys. XXIX, 253, (1966).

[11] A.H.Castro Neto and A.O.Caldeira, Phys.Rev. A42, 6884, (1990).

[12] W.H.Louisell, Quantum Statistical Properties of Radiation, (John Wiley, New York, 1973). 